\title{
Comparison of Parietal Pleural Biopsies by Rigid Forceps and Cryoprobe during Medical Thoracoscopy in Patients with Exudative Pleural Effusion
}

\author{
REFAAT A. ABO ELSAAD, M.D.*; ISMAIL M. ELWAKIL, M.D.*; AL SAYED M. TEALEB, M.D.** and \\ OMAR ABD EL-KHALEK MOHAMMED ABO SHEIR, M.Sc.*
}

The Departments of Chest Diseases* and Pathology**, Faculty of Medicine, Al-Azhar University

\begin{abstract}
Background: Medical thoracoscopy is performed under local anesthesia and conscious sedation. It has a very high diagnostic yield with fewer complications and offers opportunity to perform concurrent pleurodesis.

Aim of Study: The aim of this prospective study is to compare thoracoscopically obtained parietal pleural biopsies by rigid forceps and cryoprobe biopsies during medical thoracoscopy in patients with exudative pleural effusion.

Patients and Methods: The study included 50 patients have undiagnosed exudative pleural effusion (clinically, laboratory and radiologically); all were admitted in Al-Hussein University Hospital in Chest Department, fifty patients were implemented to medical thoracoscope after written consent.

Results: Comparing between Rigid forceps and Cryoprobe biopsies as regard number of biopsies revealed highly statistical significant difference ( $p$-value $<0.001$ ) between Rigid forceps and Cryoprobe biopsies as regard number of biopsies (170 Rigid forceps, 96 Cryoprobe). The most common definitive diagnostic results were malignant mesothelioma (32) 64\% (26 Epithelial type 52\%, 6 dysmoplastic type 12\%).
\end{abstract}

Conclusion: Pleural cryobiopsies through rigid thoracoscopy is a simple and safe procedure. It has a high diagnostic yield similar to rigid forceps biopsy. Biopsies using cryoprobe are now widely used in interventional pulmonology.

Key Words: Parietal pleural biopsies - Rigid forceps and cryoprobe - Medical thoracoscopy - Exudative pleural effusion.

\section{Introduction}

MEDICAL thoracoscopy in rigid and in semirigid technique is an efficient and safe procedure in patients with exudative pleural effusion of unknown origin [1].

Correspondence to: Dr. Omar A.M. Abo Sheir, E-Mail: dr.omar.abdelkhalek@gmail.com
Biopsy specimen taken during semi-rigidthoracoscopy is smaller than biopsies taken by rigid forceps but the diagnostic accuracy is said to be similar [2]

Cryotechnique was introduced as early as 1968 , at first for the therapeutic management of airway diseases [3].

Since then and especially in the last ten years the use of cryotechnique has been established as a routine procedure in bronchoscopy for diagnostic and interventional therapeutic use [4].

Recently, an article describing the feasibility of cryotechnique in medical, semi-rigid thoracoscopy in fifteen patients with exsudative pleural effusion was published [5].

\section{Patients and Methods}

This study was conducted in Chest Department in Endoscopic Unit in El-Hussein University Hospital in the period from May 2018 to August 2019.

A- Study design: A prospective clinical study.

$B$ - Patients: This study was included 50 patients with undiagnosed exudative pleural effusion (clinically, laboratory and radiologically).

Inclusion criteria:

1- Patients with undiagnosed exudative pleural effusion (clinically, laboratory and radiologically).

2- Adult (age above 18 years or more).

3- Signed informed consent. 


\section{Exclusion criteria:}

1- Patients with severe bleeding tendency.

2- Sever cardiac disease.

3- Diagnosed pleural effusion.

4- Age less than 18 years.

\section{Methods:}

After obtaining a written informed consent; all patients will be subjected to the followings:

1- Full medical history.

2- Full clinical examination.

3- Laboratory investigations.

4- Imaging:

1- Plain chest and heart X-ray.

2- Chest ultrasound: Used for localization of the pleural fluid and to identify potential adhesions in the pleural space.

3- Computed tomography: A CT scan is not mandatory but can be helpful to localize abnormalities such as loculated empyema or localized lesions (tumors) of the chest wall or diaphragm.

\section{5- Interventional medical thoracoscopy:}

Preparation for thoracoscopy:

A-Explanation of the technique to the patient: This is especially important when the procedure is going to be done under local anesthesia plus intravenous analgesia, because the patient will be more confident during the exploration.

$B$ - Pre-operative fasting: It is recommended that adult patients should undergo a six-hour preprocedure fast for solids, but may drink small amounts of clear fluids until two to three hours before the procedure.

$C$ - Pre-operative recordings and cannulation: The patient's temperature, pulse, blood pressure, respiratory rate and oxygen saturations should be checked and recorded prior to the procedure. A baseline electrocardiogram may be obtained for individual patient. An intravenous cannula should be placed in the hand on the same side as the planned procedure.

\section{D- Positioning, local anaesthesia and sedation:}

\section{Positioning and monitoring:}

Position of the patient:

The patient is most commonly positioned in the lateral position with the side to be examined upper most. The clinical assessment and checking a recent chest radiograph immediately before commencing the procedure. The patient's head is rested on a pillow, with the hands positioned in front of the face. This allows clear access to both the thoracic wall and the intra-venous cannula when the patient is covered with a sterile drape. A pillow placed under the patient's chest helps to spread the contra-lateral ribs, making it easier to insert the trocar and cannula and minimizing discomfort during manipulation of the thoracoscope.

The physician usually faces the patient while the assistant is across the table behind the patient's back.

\section{Skin preparation:}

Full aseptic technique should be observed. The skin over the whole hemi-thorax of the side to be examined should be prepared with an alcoholbased skin sterilizing solution. The skin preparation should include the axilla. A sterile drape should be placed over the patient, leaving a small exposed area through which the examination is performed.

\section{Local anaesthesia and site of entry:}

The recommended site of local anesthesia and chest entry is the fourth or fifth intercostal space in the mid-axillary line, within the 'safe triangle' delineated by the anterior border of latissimus dorsi, the lateral border of pectoralis major, and above a line horizontal to the nipple in the male. Thoracic ultrasound may be used immediately prior to the procedure to identify the safest and most appropriate site for trocar insertion. Avoiding areas of lung adhesion to the chest wall, local anesthesia is induced at the selected site of the procedure using up to $20 \mathrm{mls}$ of lidocaine (lidocaine) $0.5-1 \%$. The dose of infiltrated lidocaine should not exceed $3 \mathrm{mg} / \mathrm{kg}$ body weight to avoid toxicity.

An intra-dermal anesthetic bleb should initially be raised, and the intercostal muscles and parietal pleura then infiltrated with local anesthetic, aspiration of pleural fluid should be confirmed before proceeding further, unless ultrasound confirms deeper pleural effusion.

The-conscious sedation-technique or light anesthesia is simple and has practically no contraindications. The technique combines local anesthesia and neuroleptic analgesia with modern drugs that combine properties of sedation, analgesia and amnesia can be used. Suitable neuroleptics analgesia include midazolam, propofol, pethidine, diazepam and fentanyl.

\section{Performance of medical thoracoscopy/pleuroscopy:}

The patient's skin is prepared by shaving and disinfecting a large area to include from the sternum to the clavicle and across the axilla past the scapula 
to the spinous processes, and down to the base of the thorax. Then the patient is covered with sterile sheets. thoracoscopist faces the patient during the procedure (but may change position if needed), while the assistant is across.

Then the following steps are taken: At the selected point of entry (usually near the midaxillary line), a vertical incision is made with the scalpel through the skin and subcutaneous tissue, appropriate to the size of the trocar to be used, usually of approximately $10 \mathrm{~mm}$, parallel with and in the middle of the selected intercostal space. Then the trocar is inserted in a corkscrew motion until the sudden release of resistance (after passing the costal pleura) is felt, while holding the handle of the trocar firmly in the palm of the hand, as the extended index finger, for safety's sake, limits the depth of insertion needed to reach the pleural space, previously established with the local anaesthetic needle.

While the trocar is in the pleural cavity, the trocar is removed and the cannula should lie 1-3 $\mathrm{cm}$ within the pleural cavity and be held in position by the assistant. Then the thoracoscope is placed in the cannula and advanced into the pleural cavity under direct vision through the trocar. If necessary, the pleural fluid is removed with a suction catheter. In cases of a large pleural effusion, the fluid should be aspirated completely and not too hastily. This is without risk of development of immediate reexpansion oedema, as long as air is allowed to enter the pleural space through the cannula to replace the aspirated volume, thus maintaining normal intrapleural pressure. The pleural space can be inspected through the thoracoscope/ pleuroscope, either directly or indirectly by video. The endoscope is advanced towards the back and directed towards the diaphragm in the costophrenic angle. After completely removing the fluid, systematic exploration of the chest cavity is performed by maneuvering the thoracoscope/pleuroscope. Suspicious areas are biopsied through the working channel of the thoracoscope/pleuroscope. Multiple biopsies are necessary. If lesions are present on the parietal pleura, rather than visceral pleural lesions, these should be biopsied, thus avoiding the risk of prolonged air leak. Typically, two to five biopsies of a suspicious pleural lesion will establish the diagnosis. Sufficient quantities of tissue must be obtained.

\section{Biopsy technique:}

Biopsy of the parietal pleura will be performed in most medical thoracoscopy/pleuroscopy performed for diagnosis of undiagnosed exudative pleural effusions, before pleural biopsy the rib and intercostal space should be identified with a blunt probe, if the pleura is thick the rib will feel hard compared with the spongy intercostal space and if possible biopsies should always be taken against a rib to minimize the risk of vessel or nerve injury.

Using the optical biopsy forceps the parietal pleura is grasped and pulled towards the operator and it then pulled sideways and in a shearing motion, a strip of pleura can be isolated, sometimes large specimens several centimeters long can be obtained in this way.

Medical thoracoscopy with rigid single-portof-entry technique was performed in the endoscopy suite under local anesthesia and sedation as described elsewhere. All procedures were performed using a rigid medical thoracoscope $(11 \mathrm{~mm}$, Storz, Tuttlingen, Germany).

Under direct vision with the thoracoscope, all pleural fluid was removed and the pleural cavity was inspected.

6- Rigid forceps and cryoprobe biopsies techniques:

Afterwards, specimen were taken in the same patient in the same session of thoracoscopy with two methods;

Firstly biopsies were taken with Rigid forceps ( $3 \mathrm{~mm}$, Storz, Tuttlingen, Germany) and the number of biopsies were calculated, preserved in isolated bottle, Query range from (2-5) biopsies and

Lastly: Cryoprobe biopsies (2.4mm, Erbokryo CA, Erbe, Tübingen, Germany) were taken in random order, the number of biopsies were calculated, preserved in isolated bottle, Query range from (1-3) biopsies.

The cryosurgical equipment consists of the following:

The console (ERBE ELEKTROMEDIZIN, Tübingen, Germany), with line voltage 230 volt, line frequency $50 / 60 \mathrm{~Hz}$, input current $0.2 \mathrm{~A}$, line fuse T0, 2A, type no 10448-000, and serial no 11413815 . Width X height X depth was 327 X 960 $\mathrm{X} 400 \mathrm{~mm}$. Weight was $13.2 \mathrm{~kg} \mathrm{CO} 2$ cylinder was used as cryogen.

\section{Tissue sampling by cryoprobe:}

The tip of the probe, cooled to $89^{\circ} \mathrm{C}$ with $\mathrm{CO} 2$, was attached to the selected part of the parietal pleura for 30s. The frozen tissue was extracted by gently pulling.

The probe with the attached biopsy sample was removed through the thoracoscope. Each biopsy. 
Sample was released from the probe by thawing in saline and was then fixed in formalin.

\section{Post-thoracoscopic chest-tube insertion:}

At the end of thoracoscopy, a chest tube is inserted through the point of entry; it can be inserted through the cannula and guided to the required area.

To drain residual air and fluid from the pleural cavity, allowing the lung to re-expand, the tube may be quickly removed as soon as the effectiveness of the procedure is confirmed radiographically.

\section{7- Evaluation of the patients after chest tube inser-} tion:

By chest X-ray postero-anterior view after the end of thoracoscope.

\section{8-Pathological examination of samples:}

All specimens will be fixed in buffer formalin for up to 24 hours. An institutional pathologist routinely will conduct the histologic analysis for histopathological diagnosis of the 2 sample separately.

\section{Statistical analysis of data:}

Data were analyzed using Statistical Program for Social Science (SPSS) version 15.0. Quantitative data were expressed as mean \pm Standard Deviation (SD). Qualitative data were expressed as frequency and percentage.

\section{The following tests were done:}

Independent-samples $t$-test of significance: Was used when comparing between two means.

Probability ( $p$-value):

- $p$-value $<0.05$ was considered significant.

- $p$-value $<0.001$ was considered as highly significant.

- $p$-value >0.05 was considered insignificant.

\section{Results}

Table (1): Description of age in studied patients.

\begin{tabular}{cc}
\hline Variables & Studied patients $(\mathrm{N}=50)$ \\
\hline Age (years): & \\
Mean & 62.92 \\
\pm SD & 14.64 \\
Min & 23 \\
Max & 82 \\
Range & $23-82$ \\
\hline
\end{tabular}

This table shows the description of age in studied patients. The mean age of studied patients was
$62.92 \pm 14.64$ years with minimum age of 23 years and maximum age of 82 years (range 23-82).

Table (2): Descriptions of sex in studied patients.

\begin{tabular}{ccc}
\hline Variables & \multicolumn{2}{c}{ Studied patients $(\mathrm{N}=50)$} \\
\hline Sex $(n, \%):$ & & \\
Male & 30 & $60 \%$ \\
Female & 20 & $40 \%$ \\
\hline
\end{tabular}

This table shows the description of sex in studied patients. There were 30 male $(60 \%)$ and 20 females $(40 \%)$ in studied patients.

Table (3): Descriptions of side of pleural effusion in studied patients.

\begin{tabular}{lcc}
\hline Variables & \multicolumn{2}{c}{ Studied patients $(\mathrm{N}=50)$} \\
\hline Side of pleural effusion (n, \%): & & \\
Right & 34 & $68 \%$ \\
Left & 16 & $32 \%$ \\
\hline
\end{tabular}

This table shows the description of side of pleural effusion in studied patients. There were 34 patients right sided (68\%) and 16 patients left sided $(32 \%)$ in studied patients.

Table (4): Description of numbers of biopsies in studied patients.

\begin{tabular}{ll}
\hline Variables & Studied patients $(\mathrm{N}=50)$ \\
\hline Rigidforceps: & \\
Mean & 3.4 \\
\pm SD & 0.86 \\
Min & 2 \\
Max & 5 \\
Range & $2-5$ \\
Cryoprobe: & \\
Mean & 1.92 \\
\pm SD & 0.63 \\
Min & 1 \\
Max & 3 \\
Range & $1-3$ \\
\hline
\end{tabular}

This table shows the description of number of biopsies in studied patients.

- As regard rigid forceps, the mean number of biopsies in studied patients was $3.4 \pm 0.87$ with minimum number of 2 and maximum number of 5 (range 2-5).

- As regard cryoprobe, the mean number of biopsies in studied patients was $1.92 \pm 0.64$ with minimum number of 1 and maximum number of 3 (range $1-3)$. 
Table (5): Comparison between Rigid forceps and Cryoprobe biopsies as regard histopathological examination.

\begin{tabular}{|c|c|c|c|c|c|}
\hline Variables & \multicolumn{2}{|c|}{ Rigid forceps } & \multicolumn{2}{|c|}{ Cryoprobe } & $p$-value \\
\hline \multicolumn{6}{|l|}{ Histopathological diagnosis: } \\
\hline Adenocarcinoma & 2 & $4 \%$ & 2 & $4 \%$ & 1.0 \\
\hline Chronic fibrosing pleuritis with atypical mesothelial cells & 4 & $8 \%$ & 4 & $8 \%$ & \\
\hline Chronic nonspecific pleuritis & 4 & $8 \%$ & 4 & $8 \%$ & \\
\hline Malignant mesothelioma, dysmoplastic type & 6 & $12 \%$ & 6 & $12 \%$ & \\
\hline Malignant mesothelioma, Epithelial type & 26 & $52 \%$ & 26 & $52 \%$ & \\
\hline Tuberculous pleurisy & 8 & $16 \%$ & 8 & $16 \%$ & \\
\hline
\end{tabular}

This table shows no statistical significant difference ( $p$-value $>0.05$ ) between Rigid forceps and Cryoprobe biopsies as regard histopathological diagnosis.

Table (6): Description of post procedure complications in studied patients.

\begin{tabular}{lcc}
\hline Variables & \multicolumn{2}{c}{ Studied patients (N=50) } \\
\hline Post procedure complications: & & \\
No complications & 44 & $88 \%$ \\
Limited surgical emphysema & 4 & $8 \%$ \\
Trapped lung & 2 & $4 \%$ \\
\hline
\end{tabular}

This table shows the description of post procedure complications in studied patients. Trapped lung occurred in 2 patients (4\%), limited surgical emphysema occurred in 4 patients $(8 \%)$ while there were no complications in the remaining 44 patients $(88 \%)$.

Table (7): Comparison between Rigid forceps and Cryoprobe biopsies as regard number of biopsies.

\begin{tabular}{llll}
\hline Variables & $\begin{array}{c}\text { Rigid forceps } \\
(\mathrm{N}=50)\end{array}$ & $\begin{array}{c}\text { Cryoprobe } \\
(\mathrm{N}=50)\end{array}$ & $\begin{array}{c}p^{-} \\
\text {value }\end{array}$ \\
\hline Number of biopsies: & & & \\
Mean \pm SD & $3.4 \pm 0.85$ & $1.9 \pm 0.63$ & $<0.001$ \\
Sum & 170 & 96 & \\
\hline
\end{tabular}

This table shows highly statistical significant difference ( $p$-value $<0.001$ ) between Rigid forceps and Cryoprobe biopsies as regard number of biopsies.

\section{Discussion}

The present study was established to compare of parietal pleural biopsies by rigid forceps and cryoprobe during medical thoracoscopy in patients with undiagnosed exudative pleural effusion.

The study included 50 patients have undiagnosed exudative pleural effusion (clinically, laboratory and radiologically); all were admitted in $\mathrm{Al}-$ Hussein University Hospital in Chest Department, fifty patients were implemented to medical thoracoscope after written consent.
There was no significant difference between studied groups of patients regarding age and sex distribution (Tables 1,2).

These results agree with Tousheed et al. [6], there was no significant difference between studied groups of patients regarding age and sex distribution.

In the present study, we revealed side of pleural effusion in studied patients. There were 34 patients right sided (68\%) and 16 patients left sided (32\%) in studied patients. There was no significant difference between studied groups of patients regarding side of pleural effusion (Table 3 ).

These results agree with Tousheed et al. [6], there was no significant difference between studied groups of patients regarding side of pleural effusion. Comparing numbers of biopsies between two methods, there was no any significant difference in number of biopsies and histopathological diagnosis (Table 4).

In our present study, we compared between forceps biopsy and cryobiopsy of parietal pleura during medical thoracoscopy in patient with undiagnosed exudative pleural effusion.

There are more than one studies have been conducted on the efficacy and safety of cryobiopsies in pleural diseases.

Wurps et al., [7] reported use of cryobiopsy in 80 patients comparing rigid forceps, flexible forceps, and cryoprobe to obtain pleural biopsies, and showed that cryobiopsies were non inferior to flexible forceps but inferior to rigid forceps. However, for all cases they used a rigid pleuroscope, and not a semirigid pleuroscope.

In our present study cryobiopsies were obtained through a rigid pleuroscope in 50 patients with undiagnosed pleural effusions. We obtained larger sample sizes with minimal adverse effects (Table $6)$.

These results agree with Tousheed et al. [6] reported that cryobiopsies were obtained through 
a rigid pleuroscope in 87 patients with undiagnosed pleural effusions. To the best of our knowledge this is the largest series to date and also we used a longer freezing time compared with those in previous published literature. We obtained larger sample sizes without any adverse effects.

In our present study results reported that the diagnostic yield with cryobiopsy was $100 \%$ and was not significantly different from rigid biopsy as regard histopathologicaal examination (Table $5)$.

These results agree with Tousheed et al. [6], which reported that the diagnostic yield with cryobiopsy was $>98 \%$ and was not significantly different from conventional biopsy. And these results agree with Loddenkemper et al. [8] which reported that the complication rate was very low with no major bleeding or reexpansion pulmonary edema.

Ahmed et al. [9] reported that a definitive diagnosis was reached in 23 out 30 cases, with diagnostic yield of $76.6 \%$. Fifteen (50\%) cases were diagnosed as having malignancy; $14(46.7 \%)$ cases had mesothelioma, and one (3.3\%) case metastatic adenocarcinoma. Tuberculosis was found in eight (26.7\%) cases. Nonspecific inflammation was found in seven $(23.3 \%)$ cases.

In our present study results reported that 36 $(68 \%)$ cases were diagnosed as having malignancy; 26 (52\%) cases had mesothelioma, epithelial type, $6(12 \%)$ mesothelioma, dysmoplastic type and two (4\%) case metastatic adenocarcinoma. Tuberculosis was found in eight (16\%) cases. Nonspecific inflammation was found in $4(8 \%)$ cases (Table 5).

El Daboosy et al. [10] and Abd El Rehim et al. [11] reported that, malignancy was found to be the final diagnosis in most cases of undiagnosed pleural effusion this results agree with our present study and with results of Ahmed et al. [9] study.

In our present study the most common pathological type of malignancy was the malignant pleural mesothelioma, which was found in 32 patients (64\%) (Table 5).

This results agree with Ahmed et al. [9] study, which was found that the most common pathological type of malignancy was the malignant pleural mesothelioma, which was found in 14 (46.7\%) patients. This is probably owing to occupational exposure, and it is similar to other previous Egyptian studies; Helal et al. [12], Abd El Rehim et al. [11].
Other studies may show different types of prevalence, Prabhu and Narasimhan [13] ; performed medical thoracoscopy for 68 patients, and 24 patients were diagnosed as having malignant pleural effusion. Unlike our study, the commonest type was metastatic adenocarcinoma, and mesothelioma was diagnosed in only three patients.

In our present study demonstrated the feasibility and safety for obtaining biopsy specimens from parietal pleura using a cryoprobe during rigid thoracoscopy. There were minimal recorded adverse effects related to the procedure, in the present study; of post procedure complications in studied patients. Trapped lung occurred in 2 patients (4\%), limited surgical emphysema occurred in 4 patients $(8 \%)$ while there were no complications in the remaining 44 patients (88\%) (Table 6), while in Ahmed et al. [9] : No recorded adverse effects related to the procedure.

In our present study: There was no difference in diagnostic yield between cryobiopsies and regular rigid forceps biopsies. Cryotechnique was found to be easier with lesser bleeding. This was particularly true for cases with thin and highly vascular pleura. This results agree with Ahmed et al. [9]. And agree with Rozman et al. [14]: Which found that cryobiopsy samples were bigger and significantly easier for interpretation than flexible forceps biopsy samples. Diagnostic yield was the same for both techniques, and there were no bleeding problems related to the procedure.

In another study, Tousheed et al. [6], the diagnostic yield was $99 \%$ with cryobiopsy and $96 \%$ with flexible forceps biopsy. The average specimen size through cryoprobe was significantly larger than with the conventional flexible forceps, and no major complications were noted.

Wurps et al. [7] compared the two established biopsy techniques (rigid and flexible forceps biopsy) with the use of cryotechnique during medical thoracoscopy. In comparison, cryobiopsies showed a significantly larger biopsy size and depth than flexible forceps biopsies. On the contrary, rigid forceps biopsies showed significantly larger size and depth and higher diagnostic yield.

Our results are not in accordance with this study, as we found that cryobiopsy was similar to rigid forceps biopsy in term of sample size in cases of thickened pleura, and superior to rigid forceps biopsy in cases with thin highly vascular pleura, and the diagnostic yield was similar for both techniques. Rigid forceps biopsy is a well-established tool used through rigid thoracoscope, and cryotech- 
nique has proved to be superior to flexible forceps using semirigid thoracoscope.

The question raised in our study was the potential benefits that can be added when performing cryobiopsies through rigid thoracoscopy.

Our conclusion is that pleural cryobiopsies through rigid thoracoscopy is a simple and safe procedure. It has a high diagnostic yield similar to rigid forceps biopsy.

Biopsies using cryoprobe are now widely used in interventional pulmonology.

\section{Conclusion:}

Pleural cryobiopsies through rigid thoracoscopy is a simple and safe procedure. It has a high diagnostic yield similar to rigid forceps biopsy.

Biopsies using cryoprobe are now widely used in interventional pulmonology.

\section{References}

1- ROZMAN A., CAMLEK L., MARC-MALOVRH M., et al.: Rigid versus semi-rigid thoracoscopy for the diagnosis of pleural disease: A randomized pilot study. Respirology, 18: 704-10, 2013.

2- NATTUSAMY L., MADAN K., MOHAN A., et al.: Utility of semi-rigid thoracoscopy in undiagnosed exudative pleural effusion. Lung India, 32: 119-26, 2015.

3- SHESKI F.D. and MATHUR P.N.: Endoscopic treatment of early stage lung cancer. Cancer Control, 7: 35-44, 2000.

4- HETZEL J., EBERHARDT R., HERTH F.J., et al.: Cryobiopsy increases the diagnostic yield of endobronchial biopsy: A multicentre trial. Eur. Respir. J., 39: 685-90, 2012.
5- ROZMAN A., CAMLEK L., MARC-MALOVRH M., et al.: Feasibility and safety of parietal pleural cryobiopsy during semirigid thoracoscopy. Clin. Respir. J., doi: 10. 111 1/crj. 1225, 2014.

6- TOUSHEED S.Z., MANJUNATH H.P., MOHAN M.V., et al.: Cryobiopsy of the Pleura An Improved Diagnostic Tool. Journal of Bronchology and Interventional Pulmonology, 1: 37-41, 2018.

7- WURPS H., SCHONFELD N., BAUER T.T., et al.: Intrapatient comparison of parietal pleural biopsies by rigid forceps, flexible forceps and cryoprobe obtained during medical thoracoscopy: A prospective series of 80 cases with pleural effusion. BMC Pulm. Med., 16: 98-203, 2006.

8- LODDENKEMPER R., LEE P., NOPPEN M., et al.: Medical thoracoscopy/pleuroscopy: Step by step. Breathe, 2: 157-66, 2011.

9- AHMED M., SAMAR H., AMR M., et al.: Evaluation of safety and diagnostic yield of pleural cryobiopsies during thoracoscopy, Egyptian Journal of Bronchology, 13: 636, 2019.

10- EL DABOOSY M., EL HAMLY M., HALIMA K.M., et al.: Medical video assisted thoracoscopy-minimally invasive diagnostic tool for diagnosis of undiagnosed pleural effusion. Egypt J. Chest Dis. Tuberc., 62: 121-6, 2013.

11- ABD EL REHIM I.Y., MORSI A.F., EL-SHABRAWY M., et al.: The role of medical thoracoscopy in the diagnosis of exudative pleural effusion at the Chest Department of Zagazig University Hospitals. Egypt J. Bronchol., 10: 225-31, 2016

12- HELALA L., EL-ASSAL G., FARGHALLY A., et al.: Diagnostic yield of medical thoracoscopy in cases of undiagnosed pleural effusion in Kobri El-Kobba Military Hospital. Egypt J. Chest Dis. Tuberc., 63: 629-34, 2014.

13- PRABHU V. and NARASIMHAN R.: The role of pleuroscopy in undiagnosed exudative pleural effusion. Lung India, 29: 128-30, 2012.

14- ROZMAN A., CAMLEK L., MALOVRH M.M., et al.: Feasibility and safety of parietal pleural cryobiopsy during semirigid thoracoscopy. Clin. Respir. J., 10: 574-8, 2016. 


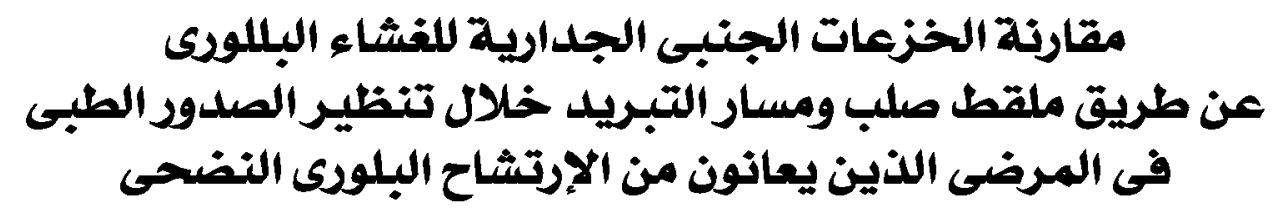

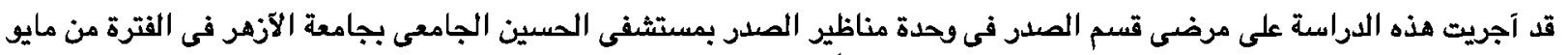

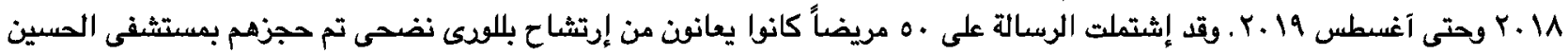

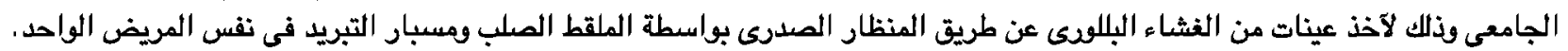

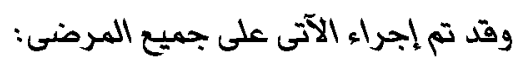

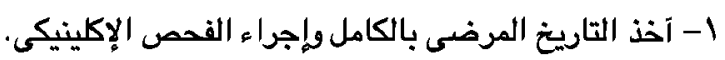

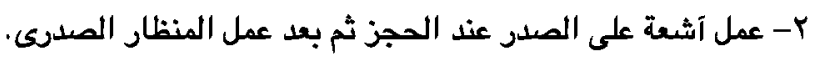

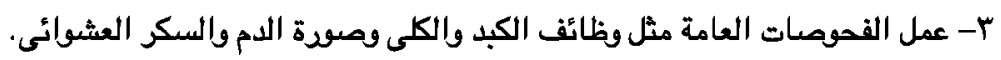
ع- عمل غازات بالدم الشريانى بصودة دورية. 0- عمل آشعة مقطعية على الصدر عند الحاجة إليها. 1- عمل آشعة تليفزيونية على الصدار قبل عمل المنظار الصدرى وذلك المساعدة فى تصديد الإختلافات فى الغشاء البللورى.

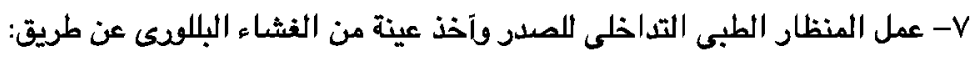

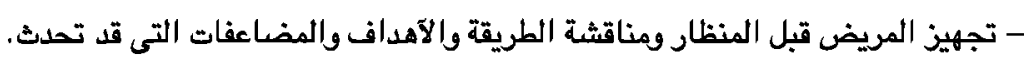

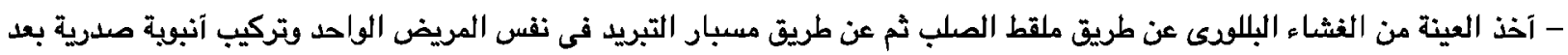

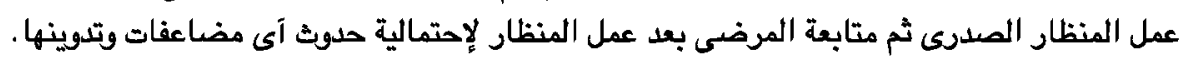

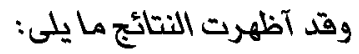

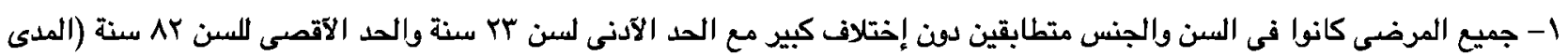

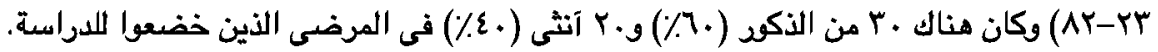


المرضى الذين شملتهم الدراسة.

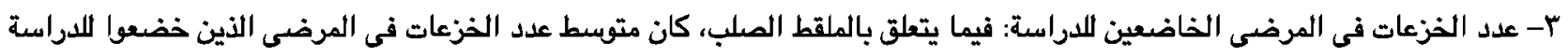

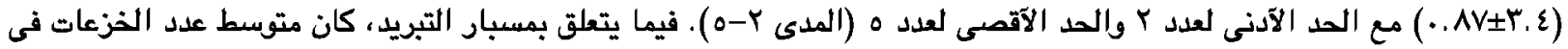

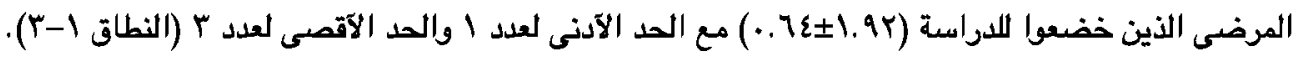

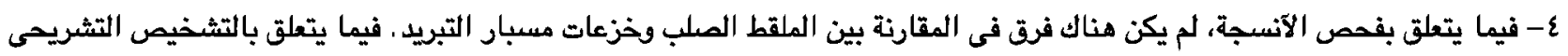
المرضى.

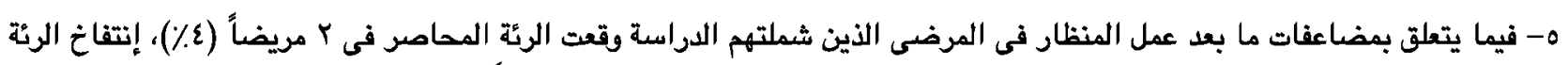

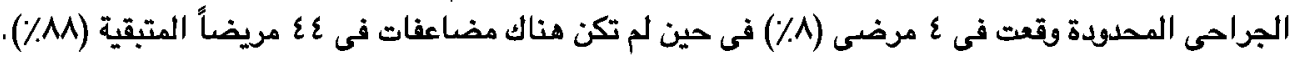

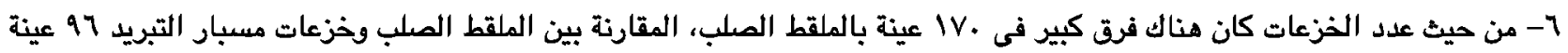
بمسبار التبريد.

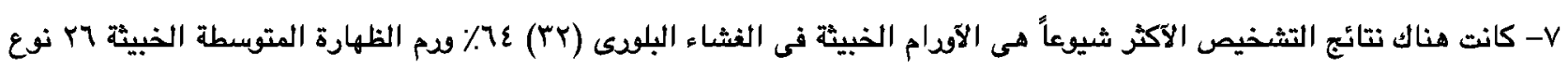

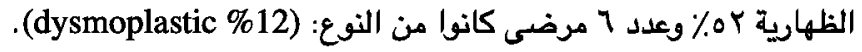

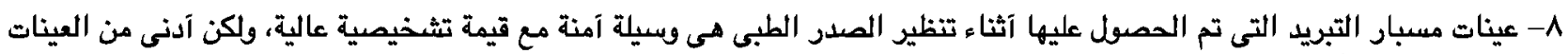
أصغر وآتّل عمقاً من خزعات الملقط الملب. خزعات ملقط الملبة. 